\title{
APROXIMACIÓN A LA FORMULACIÓN DE UN MODELO TEÓRICO DE LAS UNIDADES DE GESTIÓN DEL PAISAJE
}

approach to the formulation of a theoretical model for landscape management units

\author{
Sergio Sigarreta Vilches*
}

\begin{abstract}
Resumo
A gestão da paisagem objetiva a otimização da utilização dos bens e serviços constituidores das paisagens. Na prática pode ser entendida como a concretização dos resultados do processo de planejamento e compreende três direcionamentos principais: o uso dos potenciais não aproveitados, a manutenção de paisagens otimizadas e o ajuste das disfunções existentes nas paisagens degradadas e sua reabilitação. O surgimento e desenvolvimento do paradigma da sustentabilidade implica na apresentação de diferentes objetivos à ciência moderna e, em especial, às relacionadas ao planejamento e a gestão da paisagem. Um desses desafios constitui a geração das bases teóricas e metodológicas para a obtenção dos requisitos à sustentabilidade, que no caso da gestão da paisagem ainda se encontram pouco desenvolvidos. Este artigo se coloca em relação a esta questão e aborda alguns fatores que se pode considerar na formulação de modelos teóricos das unidades de gestão da paisagem, tais como: o geoecológico, que reflete sobre a base natural, o antropogênico, que trata das modificações e adequações culturais, o estado geoecológico atual da paisagem e estado desejado. Como resultado se apresentam os elementos essenciais que caracterizam cada um dos fatores que intervêm na configuração das unidades de gestão da paisagem e uma aproximação à expressão gráfica do modelo teórico.
\end{abstract}

Palavras chave: Paisagem, Gestão de paisagem, Modelo Teórico.

\section{Abstract}

The landscape management focal objective is to optimize the utilization process of goods and services provided by them. It could be understood, in practice, as the implementation of the planning process of landscape and have three main directions: the use of unconsidered potentials, the maintenance of the optimized landscapes state and finally the adjustment of the existent disfunctionality in degraded landscapes and its rehabilitation. The sustainability paradigm development has implied important challenges for modern science, and in particular for those related with planning and management of landscapes. One of these challenges is the development of the theory and methodological bases for the achievement of the sustainability requirements; which in the case of the landscapes management are still insufficiently developed. This paper is well-adjusted within this context and it approaches some factors that might be taken into account for the formulation of a theoretical model of the landscape management units, such as: the geoecological characteristics that reflect the landscapes natural bases; the anthropogenic factors, that shows the landscape cultural transformations and adaptations; its geoecological and desired state. As principals results are presented the essential elements that typify each factor involved in the configuration of the landscape management units and finally an approach to the model graphic representation.

Key words: Landscape, Landscape management, Theoretic model.

\begin{abstract}
Resumen
La gestión del paisaje tiene como objetivo central la optimización de la utilización de los bienes y servicios que proveen los paisajes. En la práctica puede ser entendida como la concreción de los resultados del proceso de planificación y comprende tres direcciones principales: el uso de potenciales no aprovechados, el mantenimiento de los paisajes óptimizados y el ajuste de las disfuncionalidades existentes en los paisajes degradados y su rehabilitación. El surgimiento y desarrollo del paradigma de la sostenibilidad ha implicado importantes retos para la ciencia moderna y en especial para las ciencias relacionadas con la planificación y la gestión del paisaje. Uno de estos desafíos lo constituye la generación de las bases teóricas y metodológicas para el logro de los requerimientos de la sostenibilidad, las que, en el caso de la gestión del paisaje están aún insuficientemente desarrolladas. Este artículo responde a este contexto y aborda algunos factores que pueden considerarse para la formulación del un modelo teórico de las unidades de gestión del paisaje, tales como: el geoecológico que refleja la base natural del paisaje, el antropogénico, que refleja las modificaciones y adecuaciones culturales del paisaje, el estado geoecológico actual del paisaje y estado deseado. Como resultados se presentan los elementos esenciales que caracterizan a cada uno de los factores que intervienen en la configuración de las unidades de gestión del paisaje y una aproximación a la expresión gráfica del modelo teórico.
\end{abstract}

Palabras clave: Paisaje, Gestión del paisaje, Modelo teórico.

(*) Doutorando em Geografia pela Universidad de La Habana - Centro de Investigaciones y Servicios Ambientales y Tecnológicos - Calle 18 Esq. Maceo Reparto El Llano. Holguín, - Cuba, Tel: (+ 53 24) 474448 - ss@cisat.cu 


\section{INTRODUCCIÓN}

Entre los retos de la ciencia del paisaje en la actualidad están los relacionados con las implicaciones teóricas y prácticas de la concepción del desarrollo sostenible y los problemas epistemológicos y metodológicos, que de ellas se derivan (DIAKONOV; MAMAI, 2008). La adecuación de la gestión a los nuevos requerimientos de la sostenibilidad (DE GROOT, 2006; WIGGERING, et al 2006) y la comprensión de las regularidades de los procesos de utilización de los bienes y servicios que proveen los paisajes y sus efectos, constituyen direcciones de investigación fundamentales.

La gestión del paisaje, como proceso concreto de utilización y transformación del espacio, es tan antigua como la civilización humana, pero su comprensión y aceptación como objeto de estudio de la ciencia del paisaje (PRATO, 2000), es relativamente reciente y guarda una estrecha relación con el surgimiento del paradigma de la sostenibilidad (MATEO 2007), el que ha propiciado además, que los fundamentos teóricos y metodológicos para el estudio de los paisajes hayan tenido un acelerado desarrollo y una amplia aplicación en la planificación.

En esta dirección de aplicación de los estudios de los paisajes ha incidido, sin lugar a dudas, la evolución del concepto de paisaje, introducido en la ciencia por Humboldt en 1849 (SHAW; OLDFIELD, 2007) y que tuvo dos momentos de desarrollo importantes en el siglo XX: los trabajos de Sauer, Berg y Troll en la primera mitad (TRESS; TRESS, 2001; SHAW; OLDFIELD, 2007) y el salto cualitativo que significó la introducción de la noción de sistemas al concepto de paisaje por Sochava en la década del 60 del siglo XX. (Mateo, 2008); ha continuado evolucionando.

Si bien en un inicio los paisajes naturales y culturales se consideraban como acepciones contradictorias, posteriormente han comenzado a entender como dos fenómenos interconectados (MATEO, 2007). En la actualidad los paisajes se conciben como "una parcela del espacio real objetivado, un producto antropológico... que cristaliza intensiones, intereses, necesidades y posibilidades" (Mateo, 2008), y que son expresión más tangible de las fuerzas civilizatorias de la humanidad en sus diferentes escalas espaciales y temporales.

Sin embargo, aunque frecuentemente se reconoce el valor teórico y práctico de los paisajes como objeto de gestión (PRATO, 2000; WOOD; HARDLEY, 2001; BLASCHKE, 2006; DE GROOT, 2006; MATEO, 2008), es insuficiente aún el nivel de instrumentación de los productos de la planificación, lo que revela una relativa desconexión entre ambos procesos. En esta realidad influye el limitado tratamiento por la ciencia de la unidad de gestión del paisaje, como categoría espacial clave para concreción de la sostenibilidad del paisaje. En este sentido, puede ser de utilidad práctica contar con un modelo teórico que permita contrastar las prácticas actuales de gestión con los requerimientos propuestos por la ciencia, a la luz del los nuevos paradigmas.

\section{RESULTADOS}

\section{Gestión del paisaje}

La gestión del paisaje puede ser definida como un proceso dirigido a la optimización de la utilización de los bienes y servicios que proveen los paisajes. Este proceso, que en la práctica puede ser entendido como la concreción de los resultados del proceso de planificación del paisaje (MANDER et al 2005; NATAHUARA, 2006; MATEO, 2008), comprende tres direcciones principales: el uso de potenciales no aprovechados, el mantenimiento de los paisajes optimizados y el ajuste de las disfuncionalidades existentes en los paisajes degradados y su rehabilitación.

La gestión del paisaje, en tanto proceso concebido y conducido por la sociedad, cuyo objeto se caracteriza por una alta complejidad, requiere de una concepción teórica y práctica sustentada en bases científicas que aseguraren una eficaz aplicación. 
La aproximación a la formulación de un modelo teórico puede conducirse a través de dos caminos: uno mediante el esclarecimiento de los factores que influyen en el objeto de estudio y dos mediante la comprensión de las características del objeto en sí (OSTASZEWSKA, 2006). En este caso se pretende abordar los factores principales que determinan la configuración espacial de las unidades de gestión del paisaje.

a) Categorías espaciales relacionadas con la gestión del paisaje

En primer lugar aparece el paisaje que según las concepciones más actuales (WOOD; HARDLEY, 2001; ANTIPOV, 2006; MATEO, 2007; OSTASZEWSKA, 2006; SCHRÖDER; SEPPELT, 2006) el paisaje es una de las categorías fundamentales de las ciencias geográficas y ecológicas y comprende dos visiones fundamentales: la del paisaje natural y la de paisaje cultural, que más que como acepciones contradictorias, han sido conceptualizados como dos fenómenos interconectados (MATEO, 2007). Una característica esencial del paisaje, es su existencia objetiva en diferentes niveles de complejidad.

La amplitud y plasticidad de la concepción actual sobre el paisaje, le confieren al enfoque paisajístico un alto nivel de factibilidad operativa para su aplicación, dado el amplio instrumental metodológico que comprende (CORRY; NASSAUER, 2005), el cual se ha desarrollado a partir de las diferentes disciplinas científicas que pueden integrarse en el mismo.

En segundo lugar, se encuentra la unidad de planificación del paisaje, la cual puede ser entendida como un territorio que es objeto de un ejercicio de planificación, a los fines de determinar sus potencialidades para satisfacer determinadas demandas sociales y las estrategias e instrumentos para su óptima utilización. (BLASCHKE, 2006; CORRY; NASSAUER, 2005; HAWKINS; SELMAN, 2002; MANDER et al, 2005; NATUHARA, 2006). Las unidades de planificación son la base espacial del proceso de planificación del paisaje y como tal, tienen un carácter práctico y dependen esencialmente del ámbito administrativo, es decir de la concreción de las relaciones de poder. Las mismas no responden necesariamente a estructuras definidas sobre bases científicas, ya que en su definición pueden intervenir entidades espaciales de diversa naturaleza, en correspondencia con los objetivos socio administrativos que se persigan y las escalas espaciales, por lo que pueden comprender una unidad de paisaje o un conjunto de estas.

En tercer lugar, aparece la unidad de gestión del paisaje, la cual puede concebirse como el conjunto de unidades de paisaje, establecidas sobre la base de sus propiedades y potenciales naturales y las transformaciones culturales a que han sido sometidas, que requieren de una estrategia específica, para lograr un estado ambiental deseado. Desde el punto de vista práctico, resulta sumamente importante la identificación y comprensión de las unidades de gestión del paisaje, ya que las mismas son las unidades operativas del proceso de gestión, al ser portadoras de las interrelaciones entre los procesos naturales y sociales.

Es precisamente la unidad de gestión del paisaje, la unidad espacial donde se refleja, por una parte, el rigor y la objetividad de la planificación como instrumento tecnocrático, que debe asegurar una correcta ordenación de las cargas socioeconómicas en relación con los potenciales naturales y por otra, la eficacia de la gestión del paisaje como instrumento administrativo, que debe garantizar la racionalidad de los procesos de aprovechamiento de los bienes y servicios ambientales en relación con la capacidad de los geosistemas.

\section{Factores principales que determinan las unidades de gestión del paisaje}

En la definición de las unidades de gestión del intervienen diversos factores: el geoecológico que refleja la base natural del paisaje, el antropogénico, que reflejas las modificaciones y adecuaciones culturales del paisaje, el estado geoecológico actual del paisaje y estado deseado. (Figura 1) 


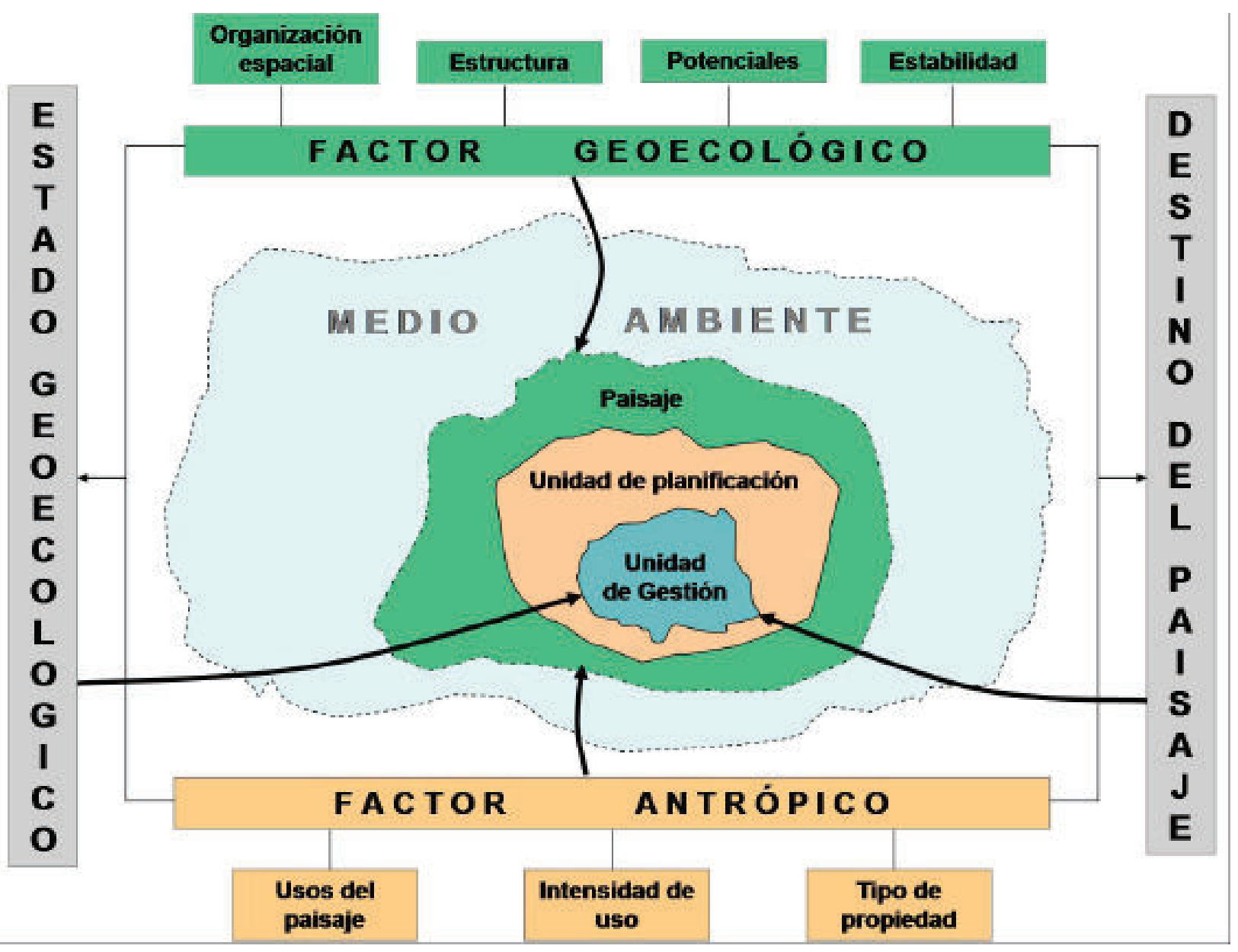

Figura 1 - Modelo teórico de las unidades de gestión del paisaje

a) Factor Geoecológico

Este factor es clave para la comprensión de los componentes y relaciones naturales que conforman la base natural del paisaje, la cual está constituida por un conjunto de componentes geográficos interrelacionados e interdependientes, gobernados por leyes naturales que operan independiente de las leyes sociales (ANTIPOV et al 2006; BASTIAN, 2006; MATEO, 2008). Para la comprensión de este factor deben considerase la organización espacial jerárquica de los paisajes, su estructura, sus potenciales de utilización y su estado geoecológico.

\section{. Organización espacial del paisaje}

Como sistema objetivo, el paisaje natural presenta diferentes niveles de organización espacial, los que se manifiestan en diferentes escalas espaciales: global, regional y local (MATEO, 2007). A los efectos prácticos de la investigación, la planificación y la gestión del paisaje, las unidades llave son: las regiones, los tipos de paisajes y los paisajes del nivel local (ACEVEDO, 1984; MATEO, 2007).

Las regiones físico - geográficas (Acevedo, 1984 y Mateo, 1984), tienen como fundamento su carácter irrepetible en el espacio y el tiempo. Es decir, la distinción y clasificación de una región, se basa en la identificación de un conjunto de paisajes que, por su unidad genética relativa y la integridad espacial, conforman una entidad que no es repetible en la geosfera, adoptando por tanto su propio nombre y una única área (ACEVEDO, 1984; CHIAPPY-JHONES et al, 2002; LIMA, 2002).

Desde el punto de vista práctico la región físico - geográfica como categoría espacial, aporta un marco referencial clave para la comprensión de la estructura y del funcionamiento del medio ambiente a escala 1:500 $000-1: 1000000$ (CHIAPPY-JHONES et al, 2002; OSINSKI, 2003; 
ANTIPOV, 2006; MATEO, 2007). Los rasgos de integridad, homogeneidad relativa y estabilidad a largo plazo propios de la región, ofrecen una ventaja significativa para planificar la sostenibilidad de la gestión del paisaje (WOOD; HARDLEY, 2001), sobre otro tipo de estructuras espaciales como las político - administrativas y las funcionales.

Al interior de cada región se manifiesta igualmente la diferenciación espacial, a través de la existencia de un conjunto interrelacionado de unidades tipológicas: tipos, clases, grupos y especies (MATEO, 1984), las cuales constituyen paisajes, tanto naturales como modificados por la actividad antrópica, que tienen como rasgo fundamental que conforman una estructura jerárquica.

En el caso de las unidades tipológicas los principios de irrepetibilidad y de integridad territorial, inherentes a la región, no se cumplen, por lo que en el marco de un mismo nivel jerárquico, las unidades presentan rasgos comunes independientemente de cuan cerca puedan encontrase, apareciendo con frecuencia distintos "parches" de un mismo tipo, clase o especie de paisaje, separados por grandes distancias.

En la práctica la profundización en la tipología de los paisajes depende del nivel de conocimiento e información disponible y pueden ser utilizadas a escalas medias y grandes (1:250 000 ó 1:100 000) para abordar la diferenciación interna de las regiones, las que se convierten en unidades de primer orden.

El carácter repetitivo de las unidades de paisaje tipológicas ofrece la posibilidad de ensayar en las mismas determinadas prácticas que luego puedan ser extrapoladas a unidades similares. Es decir que, desde el punto de vista geoecológico, es factible la posibilidad de replicar métodos, tecnologías y formas de gestión que hayan sido probados con éxito a otras unidades similares.

Los paisajes del nivel local se originan por la influencia de los procesos geográficos en espacios relativamente pequeños. Los factores que determinan la estructura espacial de los paisajes a escala global y regional, como la distribución del calor y el humedecimiento o las variaciones genéticas de las estructuras geólogo - geomorfológicas, no explican la diferenciación a escala local, que frecuentemente se caracteriza por una gran variabilidad en áreas pequeñas. En este caso se manifiesta la diferenciación morfológica o topológica de los paisajes (MATEO 1984).

La actividad humana, como factor transformador de los paisajes naturales, cobra a escala local singular relevancia. Es precisamente en este nivel de organización de la geosfera, en el que mejor se aprecian las manifestaciones del desarrollo de los procesos de aprovechamiento de los bienes y servicios ambientales, sus diferentes formas y niveles tecnológicos y sus impactos negativos. Por tanto la evaluación de los paisajes a nivel local constituye un elemento clave para la gestión de un paisaje sustentable (MANDER et al, 2005; NATUHARA, 2006), al ser portadoras de la racionalidad o la irracionalidad, de la sustentabilidad, de la huella ecológica (MATEO, 2007).

En la práctica de la gestión del paisaje, las unidades del nivel local son utilizadas a escalas grandes (1:100 000 a 1:500), lo que permite abordar diferentes niveles espaciales e instrumentos de gestión, desde los esquemas regionales hasta los proyectos ejecutivos.

\section{. Estructura del paisaje}

La estructura geoecológica es una propiedad básica de los paisajes como sistemas materiales y la misma caracteriza la forma de su organización interna, las relaciones entre los componentes que lo forman, y entre las unidades de paisajes de diferentes niveles de organización espacial y refleja los patrones organizativos existentes entre los componentes y elementos del sistema.

El análisis de la estructura permite comprender la distribución y organización jerárquica de los paisajes en el espacio geográfico y su capacidad para mantener un estado relativamente estable, ante la influencia de los impactos generados por fenómenos naturales y por procesos antropogénicos.

La estructura de los paisajes en sus diferentes niveles de organización representa la geodiversidad paisajística (MATEO, 2007) que es el resultado de la interacción dialéctica de la diversidad 
de los componentes que integran el paisaje y que constituye la base sobre la que se han desarrollado y se sostienen la diversidad biológica y la diversidad socio - cultural.

En la práctica la estructura del paisaje se convierte en un elemento clave, ya que refleja la forma en que se organiza el mismo en diferentes escalas espaciales y temporales y constituye el "QUÉ" conservar durante el proceso de gestión.

. Potenciales del paisaje

La diversidad y complejidad en la composición y la estructura de los paisajes determinan que los mismos sean portadores de una basta disponibilidad de recursos, que son susceptibles de ser aprovechados por la sociedad. Estos recursos son la base de la capacidad del paisaje (BASTIAN, 2000), para proveer bienes y servicios (RAPPORT et al, 1998; BASTIAN et al, 2006; DE GROOT, 2006; WIGGERING et al, 2006) a través de diversas funciones que permiten asegurar el desarrollo de determinados procesos socioeconómicos y culturales, la cual constituye su potencial geoecológico.

El potencial geoecológico del paisaje es un concepto que está estrechamente relacionado con el nivel de de desarrollo tecnológico y cultural de la sociedad, las demandas que éste genera y la eficiencia de los procesos de utilización de los bienes y servicios ambientales. Desde este punto de vista el paisaje cumple varias funciones, las cuales han sido agrupadas en varias categorías (modificado de GROOT, 2006):

1. Funciones de producción. Los procesos físicos, químicos y biológicos que operan en el paisaje, generan diversos recursos que son usados como bienes de consumo como la biomasa, los minerales, el agua, materiales genéticos y otros.

2. Funciones de soporte. Los recursos ambientales, crean condiciones que sostienen la vida y las actividades productivas. Por ejemplo, el agua y el aire, el suelo como hábitat natural, el suelo en las actividades agrícolas, y el agua como medio de transporte.

3. Funciones de regulación. Los procesos físicos, químicos y biológicos que operan en el paisaje limpian, acomodan, filtran, neutralizan o absorben residuos o ruidos. Estas funciones contribuyen al mantenimiento de la "salud" de los paisajes en diferentes escalas.

4. Funciones de hábitat. Los paisajes naturales y seminaturales provén refugio y condiciones propicias para la reproducción de la flora y la fauna, contribuyendo a la conservación de la diversidad genética y a los procesos de evolución de la vida silvestre.

5. Funciones socio - sicológicas. Los paisajes constituyen el marco referencial material en el que ha evolucionado la humanidad, por lo que contribuyen al mantenimiento de la salud y al desarrollo de determinados procesos sociales. En este grupo aparecen funciones tales como las cognoscitivas, informativas y recreativas

\section{. Estabilidad del paisaje}

La estabilidad geoecológica refleja la capacidad del paisaje de mantener sus propiedades sistémicas, en correspondencia con un estadio de desarrollo determinado, a la vez que mantiene vitales las funciones geoecológicas y socioeconómicas que le son inherentes, frente a los impactos naturales o antropogénicos que lo afectan.

La estabilidad geoecológica debe ser comprendida en dos direcciones. Por una parte la estabilidad natural, la cual se relaciona con la propia estructura del paisaje que determina la existencia de diferentes niveles de resistencia, elasticidad o plasticidad (MATEO, 2002) ante un mismo factor de presión externo. La estabilidad natural depende básicamente de las relaciones sinérgicas y la coherencia interna de los componentes. Por otra parte la estabilidad tecnogénica, la cual se considera como la capacidad de funcionamiento del paisaje ante los diferentes tipos de uso y su 
intensidad (MATEO, 2002). Es decir, que esta dirección se trata de comprender cómo el paisaje puede mantener sus propiedades sistémicas y el cumplimiento de sus funciones, ante los efectos de un determinado tipo e intensidad de impacto. Como base referencial empírica se parte de un tipo e intensidad de impacto ya existente o aplicado.

\section{b) Factor Antropogénico}

La comprensión del estadio actual de la evolución de los paisajes es imposible sin considerar la influencia determinante del factor antrópico. Por tanto, los paisajes contemporáneos, como categoría operativa, no son sólo el resultado de las relaciones funcionales que operan a través de los procesos naturales, sino de múltiples y complejas relaciones entre estos y los procesos socioeconómicos y culturales (BARTEL, 2000; WOOD; HARDLEY, 2001; ANTIPOV, 2006; OPDAM et al, 2006; OSTASZEWSKA, 2006; MATEO, 2007) de asimilación, ocupación y apropiación de los geosistemas y de los bienes y servicios que proveen.

El análisis de la influencia del factor antropogénico en la conformación de las unidades de gestión del paisaje, debe abordar tres elementos clave: el tipo de uso o actividad socioeconómica predominante, la intensidad del uso y el tipo de propiedad predominante.

\section{. Uso de los paisajes}

La existencia de diferentes potenciales en los paisajes para proveer bienes y servicios que contribuyen a satisfacer determinadas demandas de la Sociedad, determina la distribución espacial de los diferentes tipos de uso de los paisajes (BARTEL, 2000; NAVEH, 2001; HAWKINS Y SELMAN, 2002; CROISSANT, 2004; ANTIPOV, 2006; DE GROOT, 2006; WIGGERING et al, 2006; MATEO, 2007). En general la diversidad de usos de los paisajes se agrupa en siete tipos fundamentales: conservación, forestal, agrícolas, turístico, urbano, industrial - minero e hidráulico.

Las propias características de cada tipo de actividad socioeconómica que se desarrolla en el paisaje determinan la existencia de diferentes niveles de modificación o transformación de sus componentes y de su estructura. Cuando los cambios producidos como resultado de las actividades humanas modifican la estructura del paisaje dentro de un mismo invariante, se forma un paisaje antropo - natural. Sin embargo, si el nivel de transformación cambia la estructura y crea un nuevo invariante da como resultado el surgimiento de un paisaje antrópico (MATEO, 2002). En la medida que los procesos de transformación se intensifican y extienden espacialmente, pueden incluso provocar cambios a escala regional, que den lugar a la formación de una nueva unidad de carácter individual o región.

\section{. Intensidad del uso de los paisajes}

La intensidad del uso de los paisajes está estrechamente relacionada, en primer lugar, con el potencial de los mismos para proveer determinados bienes y/o servicios que completen el ciclo de una actividad socioeconómica concreta que surge como respuesta a determinas demandas sociales. De esta manera el potencial del paisaje define el límite que imponen las estructuras espaciales y funcionales de carácter natural a la satisfacción de cada demanda social.

En segundo lugar, se relaciona con el nivel de desarrollo tecnológico que ostenten los actores involucrados, que determina la generación, aplicación y/o aprehensión de prácticas más o menos racionales de aprovechamiento de los recursos y la disponibilidad de la técnica imprescindible para garantizar que estos procesos sean más o menos eficientes desde el punto de vista ambiental y económico.

En la práctica la intensidad del uso del paisaje frecuentemente cobra especial relevancia en el proceso de gestión del paisaje, ya que en un mismo tipo de paisaje, donde predomine un tipo específico de uso de la tierra, ésta puede determinar que se manifiesten diferencias espaciales sustanciales 
en el estado del paisaje y en la eficiencia de su utilización, requiriéndose por tanto la adopción de acciones diferenciadas, incluso dentro de la misma unidad ambiental.

\section{. Formas de propiedad}

La propiedad como categoría social tiene su esencia en la relación objeto - sujeto que se establece entre los seres humanos y su entorno material y social. La misma influye en el predominio de determinadas visiones, enfoques y conductas que determinan cómo se manifiestan en el espacio y el tiempo, las complejas interrelaciones que operan en la utilización de los potenciales de los paisajes.

Las particularidades que se aprecian en la forma de apropiación y reproducción de los bienes y servicios que proveen los paisajes, entre la forma de propiedad pública y la privada, se manifiestan en la gestión del paisaje y tienen por tanto importantes implicaciones prácticas (PRATO, 2000). En líneas generales se pueden establecer algunos ámbitos donde se manifiestan las diferencias: el tecnológico, la demanda de insumos y artificialización y la reproducción de la base que sustenta su capacidad productiva (rehabilitación, recuperación, restauración de los potenciales).

Por tanto es importante considerar los elementos antes señalados en la práctica de la gestión del paisaje, ya que los mismos pueden determinar que se manifiesten contrastes significativos en los límites de una misma unidad de paisaje.

\section{. Estado geoecológico de los paisajes}

El estado geoecológico del paisaje es una expresión de su calidad ambiental y está estrechamente relacionado con su capacidad para cumplir sus funciones naturales y proveer bines y servicios ambientales a la sociedad. El mismo puede estar determinado por: a) la relación entre colección de problemas ambientales-estado ambiental, b) eficiencia de los procesos de utilización, c) grado de modificación y transformación antopogénica, y d) vulnerabilidad o susceptibilidad del paisaje a la degradación y pérdida de su capacidad productiva.

Mateo (2002) define las siguientes clases de estado geoecológico o ambiental de los paisajes: a) Estable (no alterado): Se conserva la estructura original. No existen problemas ambientales significativos, que deterioren el paisaje. b) Medianamente estable (Sustentables): Reflejan pocos cambios en la estructura. Inciden algunos problemas de intensidad leve a moderada, que no alteran el potencial natural y la integridad del geosistema. c) Inestable (Insustentable): geosistemas que se caracterizan por fuertes cambios en la estructura espacial y funcional, de tal manera que no pueden cumplir las funciones geoecológicas, aunque aún conservan la integridad. d) Crítico: Pérdida parcial de la estructura espacial y funcional, con eliminación paulatina de las funciones geoecológicas. Se manifiesta en un significativo número de problemas ambientales de fuerte intensidad. e) Muy crítico (Catastrófico): Consiste en la pérdida y alteración generalizada de la estructura espacial y funcional de los geosistemas. El geosistema no está en condiciones de cumplir las funciones geoecológicas.

Entre el estado geoecológico, como categoría que sintetiza una situación espacio temporal específica del paisaje, y la gestión del paisaje, como proceso dirigido a aprovechar eficientemente y a mejorar las capacidades funcionales del paisaje, existe una relación biunívoca (Figura 2). En una dirección, el estado geoecológico determina el carácter espacial, temporal y funcional de la proyección estratégica y la instrumentación práctica de la gestión. En otra dirección, las intervenciones producidas por un modelo de gestión existente, determinan el estado geoecológico de los paisajes que lo sustentan y sus diferentes manifestaciones espacio - temporales. 
Determina el estado geoecológico del paisaje

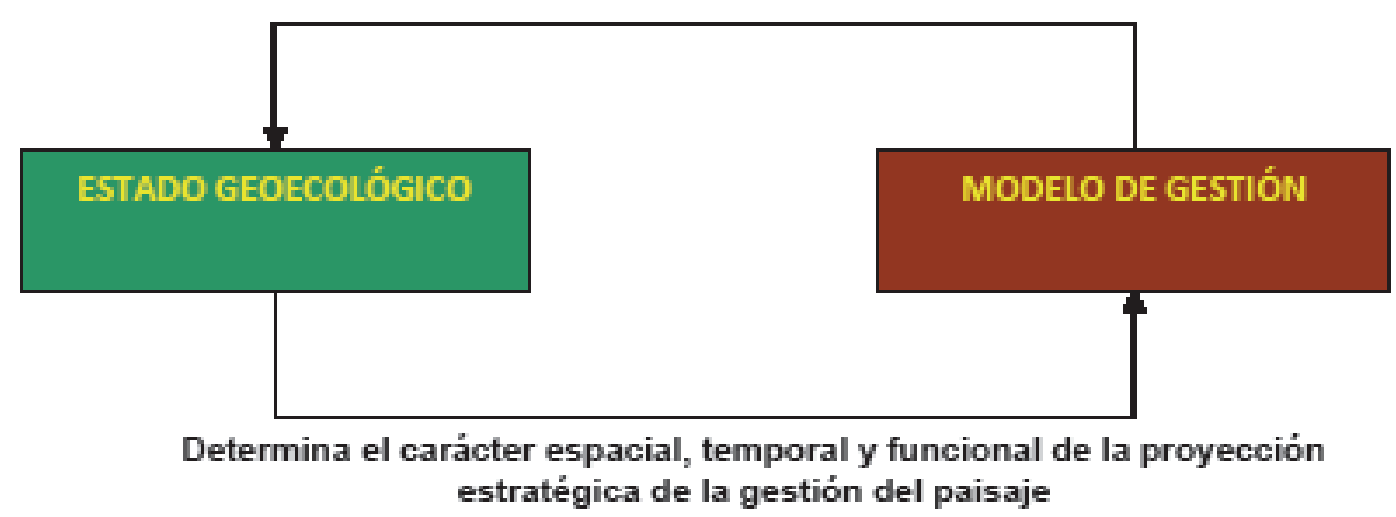

Figura 2 - Relaciones entre el estado geoecológico y el modelo de gestión del paisaje

\section{. Paisajes meta o destino}

El desarrollo de la capacidad de la Sociedad para transformar el medio ambiente hasta el límite de sus potencialidades y más, se ha convertido en un factor determinante de la situación actual de la geosfera en todos sus niveles de organización espacial. Sin embrago, el despliegue de esas mismas capacidades en función de revertir dicha situación para asegurar la sobre vivencia de la especie humana, aparece como la alternativa más viable en las próximas décadas.

En este contexto cobra especial significación la noción de paisaje meta o destino del paisaje. Sobre la base del arsenal tecnológico disponible en materia de planificación y en un ejercicio de ética socio - ambiental, que considere en su justa medida las demandas del desarrollo socioeconómico y las capacidades objetivas de los paisajes para sustentarlas, resulta relevante determinar cual es el destino óptimo (OPDAM et al, 2006) para cada paisaje.

El destino del paisaje estará en función de sus propiedades sistémicas (factor geoecológico), su utilización, su estado geoecológico y las demandas sociales de bienes y servicios previstas para un escenario específico. De manera general pueden definirse tres tipos de destinos principales que pueden asignarse al paisaje para un horizonte temporal determinado (Tabla 1).

Tabla 1 - Tipos principales de destinos del paisaje

\begin{tabular}{l|c|c|c}
\hline \multirow{1}{*}{ TIPOS } & SUBTIPO & $\begin{array}{c}\text { ESTADO GEOECOLÓGICO PREDO- } \\
\text { MINANTE }\end{array}$ & POTENCIAL DE AUTOREGENERACIÓN \\
\hline \multirow{4}{*}{ Conservación } & Áreas protegidas & Estable & Muy alto \\
\cline { 2 - 4 } & Áreas silvestres & Estable o medianamente estable & Alto \\
\hline \multirow{4}{*}{ Rehabilitación } & Restauración & Inestable o crítico & Medio \\
\cline { 2 - 4 } & Rehabilitación & Inestable & Alto \\
\cline { 2 - 4 } & Rehabilitación & Medianamente estable & Muy alto \\
\hline \multirow{4}{*}{$\begin{array}{c}\text { Producción de } \\
\text { bienes }\end{array}$} & Tutural & Estable o medianamente estable & Alto \\
\cline { 2 - 4 } & Forestal & Medianamente estable & Alto \\
\cline { 2 - 4 } & Agrícola & Medianamente estable o inestable & Alto \\
\cline { 2 - 4 } & Pecuaria & Medianamente estable o inestable & Alto \\
\cline { 2 - 4 } & Urbano & Inestable o crítico & Bajo \\
\cline { 2 - 4 } & Industrial & Crítico & Muy bajo \\
\cline { 2 - 4 } & Minero & Crítico o muy crítico & Muy bajo \\
\hline
\end{tabular}


1. Conservación. Dirigida a mantener el estado de los paisajes naturales o seminaturales a través de áreas protegidas bajo un régimen de administración o manejo. También comprende paisajes que proveen servicios ambientales evitando que se produzcan en los mismos procesos degradantes. Entre los servicios ambientales reconocidos figuran: la belleza escénica, la absorción y fijación de carbono, la fijación de metano, la investigación, la captación hídrica, la protección de suelos, la energía, la diversidad genética (banco de genes) y la producción de oxigeno

2. Rehabilitación. Dirigida a recuperar total o parcialmente la estructura o componentes del paisaje luego de procesos degradantes. Puede incluir la restauración con intervención tecnológica intensiva (conformación del microrelieve y del drenaje superficial, aporte de suelo y reforestación), la rehabilitación con intervención tecnológica de baja intensidad (reforestación) o la regeneración natural que solo implica la necesidad de que no se produzcan nuevas intervenciones degradantes para que operen los mecanismos autoregeneración del paisaje.

3. Producción. Dirigido al máximo y más racional aprovechamiento de los potenciales de proveer bienes. Incluye todas las actividades que utilizan los bienes del paisaje para satisfacer demandas sociales: turismo, forestal, agrícolas, pecuarias, urbanas, industriales y mineras.

\section{CONCLUSIONES}

La comprensión de la gestión del paisaje, como objeto de estudio, es relativamente reciente y su auge está estrechamente relacionado con dos elementos fundamentales: el desarrollo del paradigma de la sostenibilidad ambiental, y los retos que el mismo representa para la ciencia moderna y la evolución que ha experimentado el concepto de paisaje desde la segunda mitad del siglo XX.

El insuficiente nivel de instrumentación de los productos de la planificación del paisaje, revela una relativa desconexión entre este proceso y la gestión del paisaje, en lo cual puede estar incidiendo el limitado tratamiento de la unidad de gestión del paisaje, como categoría espacial clave para concreción de la sostenibilidad del paisaje.

La gestión del paisaje, en tanto proceso concebido y conducido por la sociedad, cuyo objeto se caracteriza por una alta complejidad, requiere de una concepción teórica y práctica sustentada en bases científicas. En este sentido, la formulación de un modelo teórico de las unidades de gestión del paisaje, aporta un marco referencial que permite contrastar las prácticas actuales de planificación y gestión en diferentes contextos espaciales y horizontes temporales.

En la formulación del un modelo teórico de las unidades de gestión del paisaje interviene al menos cuatro factores fundamentales: el geoecológico, el antropogénico, el estado geoecológico y el destino del paisaje o paisaje meta. Los dos primeros, además de determinar la estructura, funcionamiento y evolución del paisaje, determinan un estado específico y presuponen la construcción de un paisaje meta acorde a los condicionamientos naturales y los requerimientos culturales.

\section{BIBLIOGRAFÍA}

ACEVEDO, M. Geografía Física de Cuba. Tomo II. Editorial Pueblo y Educación. La Habana. 389 p.

ARCHER, J. H. y M. C. Jarman. Sovereign rights and responsibilities: Applying public trust principles to the management of EEZ space and resources. Ocean and Coastal Management, 17 (1), 1992. p. 251-270. ANTIPOV, A. N. (Compilador). Landscape planning: Tools and experience in implementation. Bonn Irkutsk, Russian Academy of Sciences, 2006. 345 p.

BARTEL, A. Analysis of landscape pattern: towards a 'top down' indicator for evaluation of land use. Ecological Modelling. 130, 2000. p $87-94$.

BASTIAN, O. Landscape classification in Saxony (Germany), a tool for holistic regional planning. Landscape and Urban Planning, 50, 2000. p.145-155. 
BASTIAN, O; KRONERT R.; LIPSKY Z. Landscape diagnosis on di $\square$ erent space and time scales - a challenge for landscape planning. Landscape Ecology, 21, 2006.p. 359-374.

BLASCHKE, T. The role of spatial dimension within the framework of sustainable landscape and natural capital. Landscape and Urban Planning, 75, 2006. p. 198-226.

BRITO, A. P. (Org.). Desenvolvimento Sustentável e Planejamento. Bases teóricas y conceptuais. Universidad Federal de Seará, 1997. 86 p.

CHIAPPY-JHONES, C.; GAMA, L.; SOTO-ESPARZA, M.; GEISSERT, D.; CHÁVEZ, J. Regionalización paisajística del estado de Veracruz, México Universidad y Ciencia, 18 (36), 2002. p. 87-113.

CLARK, J. R. Integrated Management of Coastal Zones. FAO Fisheries Technical Paper 327. Food and Agriculture Organization of the United Nations, Rome, 1992.

CNAP (2009). Plan del Sistema Nacional de Areas Protegidas 2009 - 2013. La Habana.

CORRY, R. C.; NASSAUER, J. Limitations of using landscape pattern indices to evaluate the ecological consequences of alternative plans and designs. Landscape and Urban Planning, 72, 2005. p. 265-280.

CROISSANT, C. Landscape patterns and parcel boundaries: an analysis of composition and configuration of land use and land cover in south-central Indiana. Agriculture, Ecosystem and Environment, 101, 2004. p. 219-232.

DE GROOT, R. Function-analysis and valuation as a tool to assess land use conflicts in planning for sustainable, multi-functional landscapes. Landscape and Urban Planning, 75, 2006. p. 75-86.

DIAJONOV, K.I.; MAMAI, I. La escuela geográfica paisajística. In: KASIMOV, N. S.- (Redactor Principal). Las escuelas científicas geográficas de la Universidad de Moscú, (en ruso); Casa Editorial Gorodiets, Moscú, 2008, 679 pgs., p. $324-386$.

HAWKINS, V.; SELMAN, P. Landscape scale planning: exploring alternative land use scenarios. Landscape and Urban Planning, 60, 2002. p. 211-224.

KLIJN, F. Ecosystem classification for enviromental management. In: Ecosystem classification for environmental Management, Kluwer Academic Publishers, Dordrecht, Holanda, 1994. 293 p.

LIMA, G. Região. A evolução de uma categoría de análise da Geografia. Boletim Goiano de Geografia, v. 22, No.1, 2002). p. 135-154.

MANDER, Ü.; MÜLLER, F.; WRBKA, T. "Functional and structural landscape indicators:Upscaling and downscaling problems. Ecological Indicators, 5, 2005. p. 267-272.

MATEO, J. Apuntes de geografía de los paisajes. ENPES, Universidad de la Habana, 1984. 408 p.

MATEO, J.; ACEVEDO, M. Regiones físico - geográficas de Cuba. In: Nuevo Atlas Nacional de Cuba, 1989.

MATEO, J. Geografía de los Paisajes. UH. Facultad de Geografía. La Habana, 2002. 192 p.

MATEO, J. Los caminos para el cambio. La incorporación de la sustentabilidad ambiental al proceso de desarrollo. La Habana, 2003. 341 p.

MATEO, J; NACIMENTO, J. La Sostenibilidad Ambiental Urbana desde una Perspectiva Espacial. Temas, No. 48, 2006. pp $66-71$.

MATEO, J. Aportes para la formulación de una teoría geográfica de la sostenibilidad. Tesis presentada en opción al Grado Científico de Doctor en Ciencias. Universidad de La Habana, 2007. 196 p.

MATEO, J. Planificación ambiental, Editorial Felix varela. La Habana, 2008. 166 p.

NATUHARA, Y. Landscape evaluation for ecosystem planning. Landscape Ecol Eng, 2, 2006. p. 3-11.

NAVEH, Z. Ten major premises for a holistic conception of multifunctional landscape. Landscape and Urban Planning. 57, 2001. p. 269-284

OPTAN, P., STEINGROVER, E.; ROOIJ, Sabine van. Ecological networks: A spatial concept for multi-actor planning of sustainable landscapes. Landscape and Urban Planning, 75, 2006. p. 322-332

ORDOÑEZ, S. Presentación de Retos y perspectivas de la Gestión del Paisaje en Canarias. M. Simancas y A. Cortina (Ed itores). Universidad Internacional Menéndez Pelayo, 2010.

OSINSKI, E. Operationalisation of a landscape-oriented indicator. Agriculture, Ecosystems and Environment, 98, 2003. p. 371-386. 
OSTASZEWSKA, K. Models of landscape units -utopia or necessity. Miscellanea geographica, 12, 2006. p. 5-11.

PÉREZ, N. Diferenciación del Ambiente Urbano en la Ciudad de La Habana. Potencialidades para la Gestión Ambiental Comunitaria. Tesis (Candidato a Doctor). - Universidad de La Habana, 1995.

PNUMA. Directrices para una Planificación y un Manejo Integrados de las Áreas Costeras y Marinas en la Región del Gran Caribe. Programa Ambiental del Caribe del PNUMA, Kingston, Jamaica, 1996. 136 p. PRATO, T. Multiple attribute evaluation of landscape management. Journal of Environmental Management, 60, 2000. p. 325-337.

SHAW, Denis J. B.; Oldfield, J. Landscape Science: A Russian Geographical Tradition, Annals of the Association of American Geographers, 97: 1, 2007. p. 111-126.

SCHRÖDER, B.; SEPPELT, R. Analysis of pattern-process interactions based on landscape models-Overview, general concepts and methodological issues. Ecological modelling, 199, 2006. p. 505-516.

SHVEVS, G.I.; SHISHENKO, P. G.; GRADZINSKII, M. D. Tipos de estructuras territoriales paisajísticas (en ruso). In: Geografía Física y Geomorfología. Editorial de la Escuela Superior, Kiev, Ucrania, 1986. p. 110-114.

SOSA, N. M. Perspectiva ética. Colección Monografías de Educación Ambiental., Fundación Universidad - Empresa. UNED, Madrid, 1995. 67 p.

TAKEUCHI, K. Landscape Planning Methodology Based on Geoecological Land Evaluation. Geo Journal 7(2): 1, 1983. p. 67-183.

TRESS, B.; TRESS, G. Capitalising on multiplicity: A transdisciplinary system approach to landscape research. Landscape and Urban Planning, 57, (2001). p. 143-157.

WIGGERING, H. C. Dalchowa; GLEMNITZ, M.; HELMING, K.; MÜLLER, Klaus; SCHULTZ, A.; STACHOWA, U.; ZANDER, P. Indicators for multifunctional land use-Linking socio-economic requirements with landscape potentials. Ecological Indicators, 6, 2006. p. 238-249.

WOOD, R.; Hardley, J. La dinámica del paisaje y la gestión del cambio. Landscape Research, 26, No.1, 2001. p. 45-54. 